\title{
The average tree solution for cooperative games with communication structure
}

Citation for published version (APA):

Herings, P. J. J., van der Laan, G., Talman, A. J. J., \& Yang, S. (2010). The average tree solution for cooperative games with communication structure. Games and Economic Behavior, 68(2), 626-633. https://doi.org/10.1016/j.geb.2009.10.002

Document status and date:

Published: 01/03/2010

DOI:

10.1016/j.geb.2009.10.002

Document Version:

Publisher's PDF, also known as Version of record

Document license:

Taverne

Please check the document version of this publication:

- A submitted manuscript is the version of the article upon submission and before peer-review. There can be important differences between the submitted version and the official published version of record.

People interested in the research are advised to contact the author for the final version of the publication, or visit the DOI to the publisher's website.

- The final author version and the galley proof are versions of the publication after peer review.

- The final published version features the final layout of the paper including the volume, issue and page numbers.

Link to publication

\footnotetext{
General rights rights.

- You may freely distribute the URL identifying the publication in the public portal. please follow below link for the End User Agreement:

www.umlib.nl/taverne-license

Take down policy

If you believe that this document breaches copyright please contact us at:

repository@maastrichtuniversity.nl

providing details and we will investigate your claim.
}

Copyright and moral rights for the publications made accessible in the public portal are retained by the authors and/or other copyright owners and it is a condition of accessing publications that users recognise and abide by the legal requirements associated with these

- Users may download and print one copy of any publication from the public portal for the purpose of private study or research.

- You may not further distribute the material or use it for any profit-making activity or commercial gain

If the publication is distributed under the terms of Article $25 \mathrm{fa}$ of the Dutch Copyright Act, indicated by the "Taverne" license above, 


\title{
The average tree solution for cooperative games with communication structure
}

\author{
P.J.J. Herings ${ }^{\mathrm{a}, *}$, G. van der Laan ${ }^{\mathrm{b}}$, A.J.J. Talman ${ }^{\mathrm{c}}$, Z. Yang ${ }^{\mathrm{d}}$ \\ a Department of Economics, Maastricht University, 6200 MD Maastricht, The Netherlands \\ ${ }^{\mathrm{b}}$ Department of Econometrics and Tinbergen Institute, Vrije Universiteit, 1081 HV Amsterdam, The Netherlands \\ c CentER and Department of Econometrics E Operations Research, Tilburg University, 5000 LE Tilburg, The Netherlands \\ ${ }^{d}$ Faculty of Business Administration, Yokohama National University, Yokohama 240-8501, Japan
}

\section{A R T I C L E I N F O}

\section{Article history:}

Received 3 September 2008

Available online 12 October 2009

\section{JEL classification:}

C71

\section{Keywords:}

Cooperative game

Graph structure

Single-valued solution

Core

Convexity

Spanning tree

\begin{abstract}
A B S T R A C T
We study cooperative games with communication structure, represented by an undirected graph. Players in the game are able to cooperate only if they can form a network in the graph. A single-valued solution, the average tree solution, is proposed for this class of games. The average tree solution is defined to be the average of all these payoff vectors. It is shown that if a game has a complete communication structure, then the proposed solution coincides with the Shapley value, and that if the game has a cycle-free communication structure, it is the solution proposed by Herings, van der Laan and Talman in 2008. We introduce the notion of link-convexity, under which the game is shown to have a non-empty core and the average tree solution lies in the core. In general, linkconvexity is weaker than convexity. For games with a cycle-free communication structure, link-convexity is even weaker than super-additivity.
\end{abstract}

(c) 2009 Elsevier Inc. All rights reserved.

\section{Introduction}

A situation in which sets of players can realize joint payoff by cooperating can be formulated as a cooperative game $(N, v)$, where $N=\{1, \ldots, n\}$ is a finite set of players and $v: 2^{N} \rightarrow \mathbb{R}$ a characteristic function with $v(S)$ the joint payoff that the players in $S \subset N$ can obtain by cooperation. In the standard approach it is assumed that any coalition $S$ can form and achieve worth $v(S)$. However, there are many situations of interest where cooperation among people depends on how they can communicate and coordinate.

In a seminal paper, Myerson (1977) formulates games with communication structure by a triple $(N, v, L)$, where $N$ is a set of players, $v: 2^{N} \rightarrow \mathbb{R}$ a characteristic function, and $L \subset\{\{i, j\} \mid i, j \in N, i \neq j\}$ a set of edges on $N$ representing communication links between players. A coalition $S$ can only cooperate if the set of nodes $S$ is connected in the graph $(N, L)$. The Myerson value of a game with communication structure equals the Shapley value of the so-called Myerson restricted game, induced by the communication structure, and is characterized by component efficiency and fairness.

Alternative characterizations of the Myerson value are given in Myerson (1980) and Borm et al. (1992). In the latter paper also another solution for games with communication structure has been proposed, the so-called positional value, see

\footnotetext{
th An earlier version of this paper was presented as "The average tree solution for cooperative games with limited communication structure" at the World Congress of the Game Theory Society, July 2008, Evanston. The first and the forth authors would like to thank The Netherlands Organisation for Scientific Research (NWO) and CentER of Tilburg University for financial support.

* Corresponding author.

E-mail addresses: P.Herings@maastrichtuniversity.nl (P.J.J. Herings), glaan@feweb.vu.nl (G. van der Laan), talman@uvt.nl (A.J.J. Talman), yang@ynu.ac.jp (Z. Yang).
} 
also Meessen (1988). This value is characterized by component efficiency and balanced total threats, see Slikker (2005). Recently, Herings et al. (2008) introduced a new solution for the class of games with cycle-free communication structure, the so-called average tree solution. This solution is characterized by component efficiency and component fairness. The average tree solution lies in the core if the characteristic function $v$ is superadditive. This property does not hold for the Myerson value and the positional value.

In this paper we generalize the average tree solution to the class of all games with communication structure. A tree $(N, T)$ is a cycle-free directed graph, with $T$ a collection of $n-1$ directed edges, such that for exactly one node, the root, there exists a unique directed path in $(N, T)$ to every other node. To generalize the average tree solution to the class of all games with communication structure, we define for every graph $(N, L)$ a collection of admissible spanning trees on the graph. A spanning tree is admissible if each player has exactly one successor in each component of his subordinates. The payoff of a player in a given admissible spanning tree is then the marginal contribution of that player when he joins his subordinates, and the proposed average tree solution is the average of the payoff vectors for all admissible spanning trees. We show that for games with cycle-free communication structure the average tree solution coincides with the average tree solution in Herings et al. (2008) and that for games with complete communication structure the average tree solution coincides with the Shapley value.

We also introduce the notion of link-convexity for games with communication structure. For games with complete communication structure, the notion of link-convexity coincides with convexity, but in general the notion of link-convexity is weaker than convexity. For games with cycle-free communication structure, link-convexity is even weaker than superadditivity. It is well known that for convex games the Shapley value lies in the core and so the Myerson value lies in the core of the game when the (restricted) game is convex. We show that for arbitrary games with communication structure, the average tree solution is in the core if the game is link-convex. This confirms the result of Herings et al. (2008) for a game with cycle-free communication structure that the average tree solution is in the core if the game is superadditive. We further illustrate that the Myerson value may not be in the core if the game is link-convex but not convex.

We notice that following this study, Baron et al. (2008) define and axiomatize the average tree solution for any class of spanning trees. They prove that our set of spanning trees is the largest class of spanning trees such that the corresponding average tree solution is a Harsanyi solution.

This paper is organized as follows. Section 2 is a preliminary section on games with communication structure. In Section 3 the average tree solution for all games with communication structure is introduced. In Section 4 the classes of cycle-free and complete communication structures are discussed. In Section 5 the notion of link-convexity is introduced and it is shown that the average tree solution lies in the core if the game is link-convex. Section 6 concludes.

\section{TU-games with communication structure}

A transferable utility cooperative game with communication structure is represented by $(N, v, L)$ with $N=\{1, \ldots, n\}$ a finite set of players, $v: 2^{N} \rightarrow \mathbb{R}$ a characteristic function, and $(N, L)$ an undirected graph with node set the set of players $N$ and a set of edges $L$, being a subset of $\{\{i, j\} \mid i \neq j, i, j \in N\}$. The set $L$ stands for the collection of communication links between players.

A sequence of different nodes $\left(i_{1}, \ldots, i_{k^{\prime}}\right)$ is called a path from $i_{1}$ to $i_{k^{\prime}}$ in the graph $(N, L)$ if $\left\{i_{k}, i_{k+1}\right\} \in L$ for $k=$ $1, \ldots, k^{\prime}-1$. A coalition of players $S \in 2^{N}$ forms a network in the graph $(N, L)$ if $S$ is connected in the graph, i.e., for any $i, j \in S, i \neq j$, there is a path in $S$ from $i$ to $j$. Notice that the empty set and all singleton coalitions are networks by definition. A coalition $S$ of players is called a component in the graph $(N, L)$ if $S$ forms a network and $S$ cannot form a larger network with any other player $j \in N \backslash S$. A sequence of at least three different nodes $\left(i_{1}, \ldots, i_{k^{\prime}}\right)$ is called a cycle in the graph $(N, L)$ if $(1)$ it is a path in $(N, L)$ and $(2)\left\{i_{k^{\prime}}, i_{1}\right\} \in L$. A graph $(N, L)$ is cycle-free if it does not contain any cycle. For given graph $(N, L)$, each $K \in 2^{N}$ induces the subgraph $(K, L(K))$, with $L(K)=\{\{i, j\} \in L \mid i, j \in K\}$ the set of links on $K$. For $K \in 2^{N}$, let $C^{L}(K)$ denote the collection of all networks in the graph $(K, L(K))$ and let $\widehat{C}^{L}(K)$ denote the collection of all components in $(K, L(K))$.

A directed graph on $N$ is a pair $(N, D)$ such that $D \subset\{(i, j) \in N \times N \mid i \neq j\}$ is a collection of directed edges. A player $i$ is a predecessor of $j$ and $j$ a successor of $i$ in $D$ if $(i, j) \in D$. A sequence of different nodes $\left(i_{1}, \ldots, i_{k^{\prime}}\right)$ is called a directed path from $i_{1}$ to $i_{k^{\prime}}$ in the directed graph $(N, D)$ if $\left(i_{k}, i_{k+1}\right) \in D$ for $k=1, \ldots, k^{\prime}-1$. A tree $(N, T)$ is a directed graph, with $T$ a collection of exactly $n-1$ directed edges, such that from exactly one node, called the root, there is a unique directed path to every other node. A player $j$ is a subordinate of $i$ in $T$ if $T$ contains a directed path from $i$ to $j$. Given an undirected graph $(N, L)$, a tree $(N, T)$ is a spanning tree of $(N, L)$ if $(i, j) \in T$ implies $\{i, j\} \in L$, i.e., any directed edge in $T$ is an undirected edge in $L$.

In the game $(N, v, L)$, a coalition $S$ of players can only cooperate and realize the worth $v(S)$ if $S$ forms a network. In the rest of the paper, we assume without loss of generality that $N$ is connected, so $N$ itself forms a network and can realize its worth $v(N)$. Otherwise, the analysis can be done analogously for each component in the graph $(N, L)$. When each pair of players can communicate, i.e., $L=\{\{i, j\} \mid i \neq j, i, j \in N\},(N, v, L)$ is said to be a game with complete communication structure and often denoted by $(N, v)$.

A payoff vector $x \in \mathbb{R}^{n}$ of $(N, v, L)$ is an $n$-dimensional vector giving a payoff $x_{i} \in \mathbb{R}$ to every player $i \in N$. We write $x(S)=\sum_{i \in S} x_{i}$ for $S \in C^{L}(N)$. A payoff vector $x$ is efficient if $x(N)=v(N)$. A solution for games with communication structure 
is a mapping $F$ that assigns to every game with communication structure $(N, v, L)$ a set of payoff vectors $F(N, v, L) \subset \mathbb{R}^{n}$. A solution $F$ is efficient if for any $(N, v, L)$ every element of $F(N, v, L)$ is efficient.

The best-known set-valued solution for games $(N, v)$ is the core, see Gillies (1953), which assigns to every game ( $N, v)$ the set $C(N, v)=\left\{x \in \mathbb{R}^{n} \mid x(N)=v(N)\right.$, and $x(S) \geqslant v(S)$, for all $\left.S \in 2^{N}\right\}$ of undominated efficient payoff vectors. For games with communication structure $(N, v, L)$ the core becomes equal to the set $C(N, v, L)$ given by

$$
C(N, v, L)=\left\{x \in \mathbb{R}^{n} \mid x(N)=v(N) \text {, and } x(S) \geqslant v(S) \text {, for all } S \in C^{L}(N)\right\} .
$$

It holds that $C(N, v, L)$ is equal to $C\left(N, v^{L}\right)$, where $v^{L}$ is the characteristic function of the Myerson restricted game $\left(N, v^{L}\right)$ induced by $(N, v, L)$ and defined for $S \in 2^{N}$ by $v^{L}(S)=\sum_{K \in \widehat{C}^{L}(S)} v(K)$.

The best-known single-valued solution for games $(N, v)$ is the Shapley value, see Shapley (1953), which assigns to every game $(N, v)$ the average $\phi(N, v)$ of all $n$ ! marginal vectors $m^{\pi}(v) \in \mathbb{R}^{n}$ of the game $(N, v)$, where $\pi=(\pi(1), \ldots, \pi(n))$ is a permutation $\pi: N \rightarrow N$ assigning a unique number $\pi(i) \in N$ to every player $i \in N$ and $m^{\pi}(v)=\left(m_{1}^{\pi}(v), \ldots, m_{n}^{\pi}(v)\right)$ with, for every $j \in N, m_{j}^{\pi}(v)=v\left(\pi^{j} \cup\{j\}\right)-v\left(\pi^{j}\right)$ and $\pi^{j}=\{i \in N \mid \pi(i)<\pi(j)\}$. The Myerson value, see Myerson (1977), is a single-valued solution assigning to every $(N, v, L)$ the Shapley value $\phi\left(N, v^{L}\right)$ of the Myerson restricted game.

\section{The average tree solution}

To extend and generalize the average tree solution for games with cycle-free communication structure as introduced in Herings et al. (2008) to the class of all games with communication structure, first notice that when a graph $(N, L)$ is not cycle-free, not all links are needed to communicate. For a particular player $i$, every spanning tree on $(N, L)$ having player $i$ as root describes a possibility in which player $i$ is able to communicate with the other players. We only consider spanning trees in which any player is linked to just one successor in every component of the set of his subordinates. We first give the definition of an admissible $n$-tuple of coalitions.

Definition 1. For given graph $(N, L)$, an $n$-tuple $B=\left(B_{1}, \ldots, B_{n}\right)$ of $n$ subsets of $N$ is admissible if it satisfies the following conditions:

(1) For all $i \in N, i \in B_{i}$, and for some $j \in N, B_{j}=N$;

(2) For all $i \in N$ and $K \in \widehat{C}^{L}\left(B_{i} \backslash\{i\}\right)$, we have $K=B_{j}$ and $\{i, j\} \in L$ for some $j \in N$.

Condition (2) of Definition 1 states that for every $i \in N$ each component in the subgraph $\left(B_{i} \backslash\{i\}, L\left(B_{i} \backslash\{i\}\right)\right)$ is equal to $B_{h}$ for some player $h$ being linked to player $i$. The same condition also implies that every set $B_{i}$ is a network. We interpret $B_{i}$ as the set of subordinates of player $i$ together with player $i$ himself. We define the directed graph $\left(N, T^{B}\right)$ as

$$
T^{B}=\left\{(i, j) \mid B_{j} \in \widehat{C}^{L}\left(B_{i} \backslash\{i\}\right), i \in N\right\} .
$$

Lemma 1. For a graph $(N, L)$, let $B$ be an admissible $n$-tuple of coalitions. Then the following properties hold:

(1) There exists a unique player $i \in N$ such that $B_{i}=N$;

(2) For all $i, j \in N, i \neq j$, either $B_{i} \subset B_{j} \backslash\{j\}$, or $B_{j} \subset B_{i} \backslash\{i\}$, or both $B_{i} \cap B_{j}=\emptyset$ and $B_{i} \cup B_{j} \notin C^{L}(N)$;

(3) The directed graph $\left(N, T^{B}\right)$ is a spanning tree.

Proof. From Condition (1) of Definition 1 it follows that $B_{i}=N$ for some $i \in N$. By Condition (2) of Definition 1 , for every $K \in \widehat{C}^{L}\left(B_{i} \backslash\{i\}\right)$ there exists $j \in N$ such that $K=B_{j}$ and $\{i, j\} \in L$, which leads to edges $(i, j)$ of $T^{B}$. Next we continue this procedure with every $j$ chosen in the previous step for which the network $B_{j}$ is not a singleton. We proceed in this way until all remaining networks are singletons. It follows immediately that $T^{B}$ is a spanning tree, which proves (3). Observe that, for all $j \in N, B_{j} \backslash\{j\}$ is the set of subordinates of player $j$ in the spanning tree $T^{B}$. Therefore, there is a unique $i \in N$ for which $B_{i}=N$, which proves (1).

To prove (2) consider two nodes $i$ and $j$. Because $T^{B}$ is a spanning tree, either $B_{i} \subset B_{j} \backslash\{j\}$ or $B_{j} \subset B_{i} \backslash\{i\}$ or $B_{i} \cap B_{j}=\emptyset$. It remains to be shown that $B_{i} \cup B_{j}$ is not a network in the last case. Since $T^{B}$ is a spanning tree, there is $j^{\prime} \neq i, j$ such that $B_{i} \subset B_{j^{\prime}}$ and $B_{j} \subset B_{j^{\prime}}$. Let $B_{j^{\prime}}$ be the minimal set with these properties. Moreover, there is no $\left(j^{\prime}, j^{\prime \prime}\right) \in T^{B}$ such that both $B_{i} \subset B_{j^{\prime \prime}}$ and $B_{j} \subset B_{j^{\prime \prime}}$, since otherwise $B_{j^{\prime \prime}}$ is a proper subset of $B_{j^{\prime}}$. It follows that $B_{i}$ and $B_{j}$ belong to different components of $\widehat{C}^{L}\left(B_{j^{\prime}} \backslash\left\{j^{\prime}\right\}\right)$, so $B_{i} \cup B_{j}$ is not connected.

In the spanning tree $T^{B}$, the root player $i$ communicates with a subordinate $h$ in $T^{B}$ through his successor $j$ in the component $K$ of $\widehat{C}^{L}(N \backslash\{i\})$ that contains $h$. Then $B_{j}=K$ and on his turn, player $j$ communicates with his subordinates through his successors in the components in $\widehat{C}^{L}\left(B_{j} \backslash\{j\}\right)$, and so on. The following example illustrates the concept of admissible $n$-tuples and their induced spanning trees. 
Example 1. Let $(N, L)$ be given by $N=\{1,2,3,4\}$ and $L=\left\{\{1,2\},\{2,3\},\{3,4\},\{4,1\}\right.$. Consider the case where $B_{1}=N$. By Condition (2) of Definition 1, for the unique component $K=\{2,3,4\}$ of the subgraph on $\{2,3,4\}$, there exists a player $i$ such that $\{1, i\} \in L$ and $B_{i}=K$. It holds that $i=2$ or $i=4$. First, take $i=2$, then $B_{2}=\{2,3,4\}$. For the unique component $K^{\prime}=\{3,4\}$ of the subgraph on $B_{2} \backslash\{2\}=\{3,4\}$, there is a player $j$ such that $\{2, j\} \in L$ and $B_{j}=K^{\prime}$. Clearly, $j=3$ and it follows that $B_{3}=\{3,4\}$. Finally, $B_{4}=B_{3} \backslash\{3\}=\{4\}$ is a leaf. Analogously, when $i=4$ is taken, $B_{4}=\{2,3,4\}, B_{3}=\{2,3\}$, and $B_{2}=\{2\}$. So there are two admissible $n$-tuple of coalitions with $B_{1}=N$. By symmetry, for any $i \in N$ there are two admissible $n$-tuples of coalitions with $B_{i}=N$ and thus there are in total 8 admissible $n$-tuples of coalitions. The two sets of admissible $n$-tuple of coalitions with $B_{1}=N$ induce two spanning trees with player 1 as root, $T_{1}=\{(1,2),(2,3),(3,4)\}$ and $T_{2}=\{(1,4),(4,3),(3,2)\}$. Observe that there are also two other spanning trees with player 1 as root, namely $T_{3}=$ $\{(1,2),(1,4),(2,3)\}$ and $T_{4}=\{(1,2),(1,4),(4,3)\}$, but these spanning trees do not correspond to an admissible $n$-tuple of coalitions, because player 1 has two successors in component $\{2,3,4\}$ of $\widehat{C}^{L}(N \backslash\{1\})$.

For a game with communication structure $(N, v, L)$, let $\mathcal{B}^{L}$ denote the collection of all admissible $n$-tuples of coalitions $B=\left(B_{1}, \ldots, B_{n}\right)$ for the graph $(N, L)$.

Definition 2. For a game with communication structure $(N, v, L)$, the marginal contribution vector $m^{B}(N, v, L) \in \mathbb{R}^{n}$ corresponding to $B \in \mathcal{B}^{L}$ is the vector of payoffs given by

$$
m_{i}^{B}(N, v, L)=v\left(B_{i}\right)-\sum_{K \in \widehat{C}^{L}\left(B_{i} \backslash\{i\}\right)} v(K), \quad i \in N .
$$

At $m^{B}(N, v, L)$ every player $i \in N$ receives a payoff equal to the worth of network $B_{i}$ minus the total worth of the components of $\left(B_{i} \backslash\{i\}, L\left(B_{i} \backslash\{i\}\right)\right)$. With respect to the corresponding spanning tree $T^{B}$, the marginal contribution gives to every player the value of the network consisting of himself and his subordinates minus the total payoff assigned to his subordinates. Notice that a marginal contribution vector $m^{B}(N, v, L)$ is a marginal vector $m^{\pi}$ of the restricted game $\left(N, v^{L}\right)$ for any permutation $\pi$ satisfying $B_{i} \backslash\{i\} \subset \pi^{i}$ for all $i \in N$. We remark that the marginal contribution vector of a spanning tree that does not belong to the set $\mathcal{B}^{L}$ of admissible spanning trees is not guaranteed to be a marginal vector of the restricted Myerson game. Therefore the spanning trees outside $\mathcal{B}^{L}$ are less appropriate.

Definition 3 (Average tree solution). On the class of all games with communication structure $(N, v, L)$, the average tree $(A T)$ solution assigns the payoff vector $\mathrm{AT}(N, v, L)$ given by

$$
\operatorname{AT}(N, v, L)=\frac{1}{\left|\mathcal{B}^{L}\right|} \sum_{B \in \mathcal{B}^{L}} m^{B}(N, v, L) .
$$

In Baron et al. (2008) it is shown that the average tree solution can be axiomatized, and that the set $\mathcal{B}^{L}$ is the largest class of spanning trees such that the average of the corresponding marginal contribution vectors is a Harsanyi solution. In addition to the marginal vector property this also justifies our choice of the set of admissible spanning trees from a different perspective.

\section{Special cases}

In this section we discuss the average tree solution for games with cycle-free communication structure and complete communication structure.

Lemma 2. Let $(N, L)$ be a cycle-free graph. Then for every $i \in N$ there is exactly one admissible n-tuple of coalitions such that $B_{i}=N$.

Proof. For some $i \in N$, take $B_{i}=N$. Since the graph is cycle-free and connected, player $i$ is linked to exactly one player in each component of $N \backslash\{i\}$. For given $K \in \widehat{C}^{L}(N \backslash\{i\})$, let $j \in K$ be the unique player such that $\{i, j\} \in L$. Then, by Condition (2) of Definition $1, B_{j}=K$. Continuing this procedure as long as there are components consisting of more than one player, we obtain the unique admissible $n$-tuple of coalitions with $B_{i}=N$.

We show that for games with cycle-free communication structure the average tree solution coincides with the solution introduced in Herings et al. (2008) for this particular class of games. When $(N, L)$ is cycle-free, let $T^{i}, i \in N$, be the unique spanning tree with node $i$ as its root. The spanning tree $T^{i}$ determines a marginal contribution vector $m^{i}(N, v, L)$ with payoff

$$
m_{j}^{i}(N, v, L)=v\left(K_{j}^{i}\right)-\sum_{\left\{j^{\prime} \mid\left(j, j^{\prime}\right) \in T^{i}\right\}} v\left(K_{j^{\prime}}^{i}\right), j \in N,
$$


where, for $j \in N, K_{j}^{i}$ is the set of nodes consisting of $j$ and all its subordinates in $T^{i}$. The average tree solution for games with cycle-free communication structure as introduced in Herings et al. (2008) then yields the average of these $n$ marginal contribution vectors.

Theorem 1. For a game with cycle-free communication structure $(N, v, L)$ it holds that

$$
\operatorname{AT}(N, v, L)=\frac{1}{n} \sum_{i \in N} m^{i}(N, v, L) .
$$

Proof. By Lemma 2 we have that for any $i \in N$ there is a unique admissible $n$-tuple of coalitions with $B_{i}=N$. Let $B(i)$ be this $n$-tuple of coalitions. From the construction in the proof of Lemma 2, it follows immediately that the spanning tree $T^{B(i)}$ corresponding to $B(i)$ coincides with the unique spanning tree $T^{i}$ having $i$ as its root. Hence $m^{i}(N, v, L)=m^{B(i)}(N, v, L)$ for all $i \in N$.

Next we prove that for games with complete communication structure the average tree solution coincides with the Shapley value.

Lemma 3. Let $(N, L)$ be a complete graph. Then there are $n !$ admissible $n$-tuples of coalitions.

Proof. For an arbitrarily chosen player $i_{1} \in N$, we consider the collection of all admissible $n$-tuples $B$ with $B_{i_{1}}=N$. At each step $k, k=2, \ldots, n$, we take an arbitrarily chosen player $i_{k}$ in $B_{i_{k-1}} \backslash\left\{i_{k-1}\right\}$ and set $B_{i_{k}}=B_{i_{k-1}} \backslash\left\{i_{k-1}\right\}$. Since at each step, any player in the remaining set can be chosen, there are $n$ ! admissible $n$-tuples of coalitions.

Theorem 2. Let $(N, v, L)$ be a game with complete communication structure. Then the average tree solution is equal to the Shapley value of $(N, v)$.

Proof. For a game $(N, v)$, the Shapley value $\phi(N, v)$ is equal to the average of all $n$ ! marginal vectors $m^{\pi}(v)$. Let $B$ be an admissible $n$-tuple of coalitions constructed in the proof of Lemma 3. For the player $i_{k}$ chosen at step $k$, the marginal contribution $m_{i_{k}}^{B}(N, v, L)$ is equal to $v\left(B_{i_{k}}\right)-v\left(B_{i_{k+1}}\right)$, where $B_{i_{n+1}}=\emptyset$. We define a bijection between admissible $n$-tuple of coalitions $B$ and permutations $\pi$ by assigning permutation $\pi^{B}$, given by $\pi_{i_{k}}^{B}=n+1-i_{k}, k=1, \ldots, n$, to admissible $n$-tuple of coalitions $B$. By definition of the marginal vector we have that $m^{\pi^{B}}(v)=m^{B}(N, v, L)$.

\section{Core properties}

In this section we provide conditions for arbitrary games with communication structure under which the average tree solution lies in the core. For a game $(N, v)$ it is well-known that the Shapley-value $\phi(N, v)$ is in the core $C(N, v)$ if the game is convex, i.e., $v(S)+v(T) \leqslant v(S \cup T)+v(S \cap T)$ for every $S, T \subset N$. A game is superadditive if these inequalities are satisfied for all $S$ and $T$ with $S \cap T=\emptyset$. Superadditivity is insufficient to ensure that a game has a non-empty core. We say that a game with communication structure $(N, v, L)$ is superadditive if its Myerson restricted game $\left(N, v^{L}\right)$ is superadditive. It can be shown that a superadditive game with cycle-free communication structure has a non-empty core. In particular, it follows from Demange (2004) that any marginal contribution vector $m^{i}(N, v, L)$ as defined in Eq. (3) is in $C\left(N, v^{L}\right)$. In fact, when $v^{L}$ is superadditive, then $v^{L}$ is permutationally convex for any permutation corresponding to the spanning tree $T^{i}$ and then, according to Granot and Huberman (1982), $m^{i}(N, v, L)$ is in $C\left(N, v^{L}\right)$ for all $i \in N$. So, for superadditive games with cycle-free communication structure the average tree solution is in $C(N, v, L)$, because the core is convex. Also for games with cycle-free communication structure, Talman and Yamamoto (2008) provide a condition even weaker than superadditivity under which the average tree solution is still in the core. We next introduce the notion of link-convexity, which will be shown to assure that the average tree solution is an element of the core for an arbitrary game with communication structure.

Definition 4 (Link-convexity). A game with communication structure $(N, v, L)$ is link-convex if

$$
v(S)+v(T) \leqslant v(S \cup T)+\sum_{K \in \widehat{C}^{L}(S \cap T)} v(K),
$$

for any $S, T \subset N$ that satisfy

(1) $S, T, S \backslash T, T \backslash S$, and $(S \backslash T) \cup(T \backslash S)$ are non-empty networks;

(2) $N \backslash S$ or $N \backslash T$ is a network. 
Notice that Condition (1) of Definition 4 implies that $S \cup T$ is a non-empty network.

Link-convexity reduces to convexity for the class of games with complete communication structure because for those games all subsets of $N$ are networks and convexity is satisfied trivially when $S, T, S \backslash T$, or $T \backslash S$ equals the empty set. We illustrate the notion by an example.

Example 2 (Cycle graph). We consider the graph $(N, L)$ with $L=\{\{i, i+1\} \mid i=1, \ldots, n\}$, where $n+1=1$. In this case any non-empty network has form $S=[i, j]$, where $[i, j]$ denotes the set $\{i, i+1, i+2, \ldots, j\}$ if $j \geqslant i$ and $[i, j]$ denotes the set $\{i, i+1, \ldots, n, 1, \ldots, j\}$ if $j<i$. Observe that for any $S=[i, j]$, the set $N \backslash S$ is a network. By Condition (1) of Definition 4 we must have that both $S$ and $T$ are non-empty networks, so for some $i, i^{\prime}, j, j^{\prime}, S=\left[i, i^{\prime}\right]$ and $T=\left[j, j^{\prime}\right]$. Then both $N \backslash S$ and $N \backslash T$ are networks, so Condition (2) of Definition 4 is redundant. Without loss of generality assume that $j \geqslant i$. Then the condition that $S \backslash T$ and $T \backslash S$ are non-empty, requires that $j>i$. Now, if $j \neq i^{\prime}+1$ then we must have that $j^{\prime}=i-1$, otherwise $(S \backslash T) \cup(T \backslash S)$ is not a network. Therefore, for the game with cyclic communication structure the link-convexity property requires that $v(S)+v(T) \leqslant v(S \cup T)+v(S \cap T)$ for all sets $S=\left[i, i^{\prime}\right]$ and $T=\left[j, j^{\prime}\right]$ such that $j>i$ and further $j=i^{\prime}+1$ or $j^{\prime}=i-1$.

Theorem 3. If the game with communication structure $(N, v, L)$ is link-convex, then $A T(N, v, L) \in C(N, v, L)$.

Proof. Consider any $B \in \mathcal{B}^{L}$ and $S \in C^{L}(N)$. We write $m^{B}=m^{B}(N, v, L)$. We show that $\sum_{i \in S} m_{i}^{B} \geqslant v(S)$, from which it follows that $m^{B} \in C(N, v, L)$, which proves the result because the core is a convex set.

Consider the subgraph $\left(S, T^{B}(S)\right)$. It has components $S_{1}, \ldots, S_{k^{\prime}}$ which are all trees, with roots $r_{1}, \ldots, r_{k^{\prime}}$. Without loss of generality, let $r_{1}, \ldots, r_{k^{\prime}}$ be such that $k^{1}<k^{2}$ implies $B_{r_{k} 1} \subset B_{r_{k^{2}}}$ or $B_{r_{k} 1} \cap B_{r_{k^{2}}}=\emptyset$. For $k=0, \ldots, k^{\prime}$, let $B^{k}=B_{r_{1}} \cup \cdots \cup B_{r_{k}}$, so it follows that $B^{0}=\emptyset$.

For $k=1, \ldots, k^{\prime}$, those successors of $S_{k}$ in the tree $T^{B}$ that lie outside $S$ are denoted by $F_{r_{k}}$. Recall that the successors of a player are his immediate subordinates. We write $R=\left\{r_{1}, \ldots, r_{k^{\prime}}\right\}$ and $I=\bigcup_{r \in R} F_{r}$. We define a tree $T^{*}$ with root $r_{k^{\prime}}$ on the nodes in $R \cup I$, where the successors of a node $r \in R$ are given by $F_{r}$ and the successors of a node $i \in I$ are given by

$$
F_{i}=\left\{r \in R \mid B_{r} \subset B_{i} \text { and } \nexists r^{\prime} \in R \backslash\{r\} \text { such that } B_{r} \subset B_{r^{\prime}} \subset B_{i}\right\} .
$$

Consider some $k \in\left\{1, \ldots, k^{\prime}\right\}$ and write $F_{r_{k}}=\left\{i_{1}, \ldots, i_{\ell^{\prime}}\right\}$. If $F_{r_{k}}=\emptyset$, then we define $\ell^{\prime}$ to be zero. When $\ell^{\prime} \geqslant 1$, then, for $\ell=1, \ldots, \ell^{\prime}$, the two sets $S \cup B^{k-1} \cup\left(B_{i_{1}} \cup \cdots \cup B_{i_{\ell-1}}\right)$ and $B_{i_{\ell}}$ satisfy Conditions (1) and (2) of Definition 4. Notice that the components of their possibly empty intersection are the networks $B_{r}$ for $r \in F_{i_{\ell}}$ and that $B_{i_{\ell}} \backslash\left(\bigcup_{r \in F_{i_{\ell}}} B_{r}\right)$ is linked to $S_{k}$. Now it follows from link-convexity that for $\ell=1, \ldots, \ell^{\prime}$,

$$
v\left(S \cup B^{k-1} \cup\left(B_{i_{1}} \cup \cdots \cup B_{i_{\ell-1}}\right)\right)+v\left(B_{i_{\ell}}\right) \leqslant v\left(S \cup B^{k-1} \cup\left(B_{i_{1}} \cup \cdots \cup B_{i_{\ell}}\right)\right)+\sum_{r \in F_{i_{\ell}}} v\left(B_{r}\right) .
$$

By repeated application of this argument and since $S_{k} \cup B^{k-1} \cup\left(\bigcup_{i \in F_{r_{k}}} B_{i}\right)=B^{k}$, it follows that for $k=1, \ldots, k^{\prime}$,

$$
v\left(S \cup B^{k-1}\right)+\sum_{i \in F_{r_{k}}} v\left(B_{i}\right) \leqslant v\left(S \cup B^{k}\right)+\sum_{i \in F_{r_{k}}} \sum_{r \in F_{i}} v\left(B_{r}\right) .
$$

Notice that this formula is also valid if $F_{r_{k}}=\emptyset$, since then $S \cup B^{k-1}=S \cup B^{k}$.

By repeated application of the last inequality, we find that

$$
v(S)+\sum_{k=1}^{k^{\prime}} \sum_{i \in F_{r_{k}}} v\left(B_{i}\right) \leqslant v\left(S \cup B^{k^{\prime}}\right)+\sum_{k=1}^{k^{\prime}} \sum_{i \in F_{r_{k}}} \sum_{r \in F_{i}} v\left(B_{r}\right) .
$$

Since $S \cup B^{k^{\prime}}=B_{r_{k^{\prime}}}$ and $T^{*}$ is a tree, it follows that every $B_{r_{k}}, k=1, \ldots, k^{\prime}-1$, appears exactly once in the right-hand side, and we obtain

$$
v(S)+\sum_{k=1}^{k^{\prime}} \sum_{i \in F_{r_{k}}} v\left(B_{i}\right) \leqslant \sum_{k=1}^{k^{\prime}} v\left(B_{r_{k}}\right) .
$$

For $k=1, \ldots, k^{\prime}, B_{r_{k}}=S_{k} \cup\left(\bigcup_{i \in F_{r_{k}}} B_{i}\right)$, so

$$
\sum_{i \in S} m_{i}^{B}=\sum_{k=1}^{k^{\prime}}\left[v\left(B_{r_{k}}\right)-\sum_{i \in F_{r_{k}}} v\left(B_{i}\right)\right] .
$$

From Eqs. (4) and (5) it follows that $v(S) \leqslant \sum_{i \in S} m_{i}^{B}$, which completes the proof. 
Corollary 1. If a game with communication structure $(N, v, L)$ is link-convex, then the core $C(N, v, L)$ is non-empty.

For games with complete communication structure link-convexity and convexity coincide with each other. The next lemma shows that under weaker conditions than superadditivity, a game with cycle-free communication structure is linkconvex.

Theorem 4. A game with cycle-free communication structure $(N, v, L)$ is link-convex if and only if for every $S \in C^{L}(N)$ it holds that

$$
v(S)+v(T) \leqslant v(S \cup T) \text { for all } T \in \widehat{C}^{L}(N \backslash S) .
$$

Proof. Let $S, T \subset N$ satisfy the conditions in Definition 4 with $N \backslash T$ being a network. We first show that $S \cap T=\emptyset$. Suppose $S \cap T \neq \emptyset$. Take any $i^{\prime} \in S \cap T$. Since $S \backslash T$ and $T \backslash S$ are non-empty and $(S \backslash T) \cup(T \backslash S)$ is a non-empty network, there exists $i \in S \backslash T$ and $j \in T \backslash S$ such that $\{i, j\} \in L$. Since both $S$ and $T$ are networks, there exists a path in $S$ connecting $i$ and $i^{\prime}$ and there exists a path in $T$ connecting $j$ and $i^{\prime}$. This contradicts the fact that $(N, v, L)$ is a game with cycle-free communication structure.

From $S \cap T=\emptyset$, it follows that $S \subset N \backslash T$. Since both $N \backslash T$ and $S \cup T$ are networks, $S \subset N \backslash T$, and (N,L) is cycle-free, we must have $T \in \widehat{C}^{L}(N \backslash S)$.

The lemma shows that the condition $v(S)+v(T) \leqslant v(S \cup T)$ only has to hold for any network $S$ and any network $T$ that is a component of $(N \backslash S, L(N \backslash S))$. Notice that for a game with cycle-free communication structure superadditivity requires that $v(S)+v(T) \leqslant v(S \cup T)$ for any disjoint $S$ and $T$ such that $S, T$, and $S \cup T$ are networks.

Corollary 2. A game with cycle-free communication structure $(N, v, L)$ is link-convex if $v$ is superadditive.

The following example illustrates that link-convexity is strictly weaker than superadditivity and also that the Myerson value may not be in the core if the game is link-convex.

Example 3 (Path graph). We consider the cycle-free graph on $(N, L)$ with $L=\{\{j, j+1\} \mid j=1, \ldots, n-1\}$. Any non-empty network $S$ is of the form $S=[i, j], 1 \leqslant i \leqslant j \leqslant n$, where $[i, j]=\{i, \ldots, j\}$. By Theorem 4 , link-convexity requires $v(S)+$ $v(T) \leqslant v(S \cup T)$ for all coalitions $S$ and $T$ such that $S=[i, j]$ and $T=[1, i-1]$ or $T=[j+1, k]$.

Consider the path graph with $N=4$ and $L=\{\{1,2\},\{2,3\},\{3,4\}\}$. The values of the connected coalitions are given by $v(\{1\})=v(\{4\})=0, v(\{2\})=2, v(\{3\})=4, v([1,2])=v([2,3])=2, v([3,4])=4, v([1,3])=v([2,4])=6$, and $v([1,4])=6$. This game is not superadditive, since for the networks $S=\{2\}$ and $T=\{3\}$ we have that $v([2,3])=2<v(\{2\})+v(\{3\})=6$. Link-convexity only requires that

$$
\begin{aligned}
& v([1, j])+v([j+1, k]) \leqslant v([1, k]), \quad j=1,2,3, j+1 \leqslant k \leqslant 4, \\
& v([i, j])+v([j+1,4]) \leqslant v([i, 4]), \quad i=2,3, i \leqslant j<4 .
\end{aligned}
$$

All these inequalities are satisfied. Observe that this game has a unique core element $(0,2,4,0)^{\top}$.

The average tree solution for this game is equal to the average of the marginal contribution vectors of the spanning trees induced by the four admissible 4-tuples $B^{1}=(N,\{2,3,4\},\{3,4\},\{4\}), B^{2}=(\{1\}, N,\{3,4\},\{4\}), B^{3}=(\{1\},\{1,2\}, N,\{4\})$, and $B^{4}=(\{1\},\{1,2\},\{1,2,3\}, N)$. All these 4-tuples yield the same marginal contribution vector, $(0,2,4,0)^{\top}$.

To compute the Myerson value of the game, we first determine the Myerson restricted game $\left(N, v^{L}\right)$. For $S \in C^{L}(N)$ we have $v^{L}(S)=v(S)$ and for $S \notin C^{L}(N)$ we have $v^{L}(\{1,3\})=4, v^{L}(\{1,4\})=0, v^{L}(\{2,4\})=2, v^{L}(\{1,3,4\})=4$, and $v^{L}(\{1,2,4\})=2$.

The Myerson value is equal to the Shapley value of the game $\left(N, v^{L}\right)$ and therefore equal to $\left(\frac{1}{3}, \frac{5}{3}, \frac{11}{3}, \frac{1}{3}\right)^{\top}$. Notice that the Myerson value lies outside the core.

\section{Concluding remarks}

In this paper the average tree solution is proposed for the class of all games with communication structure. This solution generalizes both the solution introduced by Herings et al. (2008) for the class of games with cycle-free communication structure and the Shapley value for the class of games with complete communication structure. We introduce the condition of link-convexity under which the average tree solution is an element of the core. For the class of games with cycle-free communication structure, link-convexity is weaker than superadditivity. In general, link-convexity is weaker than convexity, and only coincides with it for games with complete communication structure.

\section{References}

Baron, R., Béal, S., Rémilla, E., Solal, P., 2008. Average tree solutions for graph games. Preprint. 
Borm, P., Owen, G., Tijs, S.H., 1992. On the position value for communication situations. SIAM J. Discrete Math. 5, 305-320.

Demange, G., 2004. On group stability in hierarchies and networks. J. Polit. Economy 112, 754-778.

Gillies, D.B., 1953. Some Theorems on $n$-Person Games. Princeton University Press, Princeton.

Granot, G., Huberman, G., 1982. The relationship between convex games and minimal cost spanning tree games: A case for permutationally convex games.

SIAM J. Algebraic Discrete Methods 3, 288-292.

Herings, P.J.J., van der Laan, G., Talman, A.J.J., 2008. The average tree solution for cycle-free graph games. Games Econ. Behav. 62, 77-92.

Meessen, R., 1988. Communication games. Master Thesis, University of Nijmegen, Nijmegen (in Dutch).

Myerson, R.B., 1977. Graphs and cooperation in games. Math. Oper. Res. 2, 225-229.

Myerson, R.B., 1980. Conference structures and fair allocation rules. Int. J. Game Theory 9, 169-182.

Shapley, L., 1953. A value for n-person games. In: Kuhn, H.W., Tucker, A.W. (Eds.), Contributions to the Theory of Games, vol. II. Princeton University Press, Princeton, pp. 307-317.

Slikker, M., 2005. A characterization of the position value. Int. J. Game Theory 33, 505-514.

Talman, A.J.J., Yamamoto, Y., 2008. Average tree solution and subcore for acyclic graph games. J. Oper. Res. Soc. Japan 51, $203-212$. 